\title{
Distributed Resource Management by Using Population Dynamics: Wastewater Treatment Application
}

\author{
J. Barreiro-Gomez, N. Quijano, Senior Member, IEEE, and C. Ocampo-Martinez, Senior Member, IEEE
}

\begin{abstract}
Industrial processes commonly involve different sub-systems, each one of them with an associated controller. Moreover, although these sub-systems might be independent, the energy that the controllers use can be obtained from a limited common source. Furthermore, the total energy used in a control process might be limited because of economical reasons. A proper management of the energy resource would be achieved by having a central decision maker for this task using information of all the control signals. However, this centralized scheme would require an expensive communication network. As an alternative, the total available energy can be divided into the controllers within the process ensuring an upper bound of energy consumption. This work shows that dividing the resource throughout the controllers is not efficient regarding the control performance. Then, a distributed strategy is proposed to manage the available energy resource by using evolutionary game theory. Finally, the proposed strategy is implemented on a wastewater treatment system that considers a first stage given by an urban drainage system (UDS), and a final stage given by a non-drinkable water network (NDWN), i.e., water for streets cleaning, plants watering, etc. Simulation results show an improvement of the performance with the proposed methodology in comparison with an off-line resource management of energy.
\end{abstract}

Index Terms-Evolutionary game theory, distributed control, distributed resource allocation

\section{INTRODUCTION}

Large industrial systems commonly involve large processes that are composed by several sub-systems. One general scheme for an industrial process is given by a sequence of sub-systems, where different variables are desired to be controlled. This scheme leads to the use of local controllers operating at different independent stages. However, although these controllers operate independently, the total energy resource, which all controllers use, is supplied by a same limited source. Besides, in an industrial application, it might be desired to limit the amount of energy that is supplied by a central generator unit due to economical policies. Some similar problems involving a total constraint in the control actions have been treated as a resource allocation problem,

J. Barreiro-Gomez and N. Quijano are with Departamento de Ingeniería Eléctrica y Electrónica, Universidad de los Andes, Carrera $1^{\mathrm{A}}$ No $18 \mathrm{~A}-10$, Colombia \{j.barreiro135, nquijano\}@uniandes.edu.co.

J. Barreiro-Gomez and C. Ocampo-Martinez are with the Automatic Control Department, Universitat Politècnica de Catalunya, Institut de Robòtica Informàtica Industrial (CSIC-UPC), Llorens i Artigas, 4-6, 08028 Barcelona, Spain. \{jbarreiro, cocampo\}@iri.upc.edu

Authors would like to thank Colciencias-Colfuturo and Agència de Gestió d'Ajust Universitaris i de Recerca AGAUR for supporting J. BarreiroGomez. Authors would also like to thank Mexichem, Colombia for partially supporting this research through the project "Drenaje Urbano y Cambio Climtico: hacia los sistemas de alcantarillado del futuro. Fase II", and the project ECOCIS (Ref. DPI2013-48243-C2-1-R), which have partially supported this work e.g., for the temperature control [7], or for the dispatch of distributed generators [8].

On the other hand, in the large-scale case, it is expensive to manage the energy in a central manner. This centralized approach would imply the need of a large communication network. Consequently, the immediate solution for this issue is to divide the available amount of energy throughout all the controllers in an off-line manner. However, this work shows that this alternative might affect considerably the performance of the controllers. This problem motivates the investment of efforts to propose control techniques that take advantage of all the available energy in a distributed way, and obtaining an enhanced control performance.

The main contribution of this paper is the application of the distributed projection dynamics already proposed in [4] to manage a resource in a distributed way for controllers working at different stages in a serial topology. This application is inspired by the work presented in [3], where the distributed Smith dynamics have been used to make noncentralized a model predictive control scheme. The proposed methodology takes the control actions computed by local controllers, and determines the proper final control action to apply to actuators, and by considering an energy constraint in a distributed manner. It is shown that when independent controllers are already tuned, then it is possible to manage a common energy resource without affecting the existing design. Moreover, three different scenarios are compared. First, the control performance without resource constraint is shown. Then, the on-line distributed resource management strategy is compared with a case in which the energy resource is divided in an off-line manner.

The reminder of this paper is as follows. Section II presents the mathematical formalism of the population dynamics, states the distributed projection dynamics, and introduces the proposed distribution resource management approach. Section III presents the wastewater treatment application within a water cycle as a case study. Afterwards, the results of the proposed approach are shown and compared with an off-line management strategy in Section IV. Finally, the concluding remarks are made in Section V.

Notation: column vectors are denoted by lower-case letters and bold style, e.g., p. Matrices are denoted by uppercase letters and bold style, e.g., A. Scalars are denoted by non-bold style, e.g., $n$. Sets are denoted by calligraphic style, e.g., $\mathcal{S}$. The column vector of $n$ unitary entries is denoted by $\mathbb{1}_{n} \in \mathbb{R}^{n}$, i.e., $\mathbb{1}_{n}=\left[\begin{array}{lll}1 & \ldots & 1\end{array}\right]^{\top}$. The cardinality of a set $\mathcal{X}$ is denoted by $|\mathcal{X}|$. The set of real numbers is denoted by $\mathbb{R}$, the set of non-negative real numbers is denoted by 
$\mathbb{R}_{+}$, and the set of strictly positive real numbers is denoted by $\mathbb{R}_{++}$. Similarly, the set of positive integer numbers is denoted by $\mathbb{Z}_{+}$.

\section{POPUlATION DYNAMICS APPROACH}

The mathematical formalism of the population dynamics approach is presented by making an analogy with a dynamical system. First of all and for clarity, Table I presents an analogy between elements in the population dynamics approach with elements in a dynamical system.

TABLE I

EQUIVALENCE BETWEEN POPULATION DYNAMICS AND CONTROL THEORY ELEMENTS

\begin{tabular}{ll}
\hline Population dynamics element & Control systems element \\
\hline \hline Population & System \\
Strategy & Actuator \\
Population mass & Total energy \\
Agent & Energy unit \\
Proportion of agents & Proportion of energy \\
Strategic distribution & Energy distribution \\
Fitness of a strategy & Energy requirements of an actuator \\
\hline
\end{tabular}

Consider a system (population) with $n \in \mathbb{Z}_{+}$sub-systems, each one with an actuator (strategy). The total available energy in the system (population mass) is denoted by $m \in$ $\mathbb{R}_{+}$. The total energy is composed of a large and finite amount of energy units (agents). Each energy unit is assigned to an actuator, where the set of actuators is denoted by $\mathcal{S}=\{1, \ldots, n\}$. The scalar $p_{i}$ is the proportion of energy units assigned to the actuator $i \in \mathcal{S}$. The vector $\mathbf{p} \in \mathbb{R}_{+}^{n}$ is the energy distribution throughout the $n$ actuators. The set of the possible distributions of energy is given by a simplex $\Delta=\left\{\mathbf{p} \in \mathbb{R}_{+}^{n}: \mathbf{p}^{\top} \mathbb{1}_{n}=m\right\}$, and the interior of the set of the possible distribution of energy is given by the set $\tilde{\Delta}=\left\{\mathbf{p} \in \mathbb{R}_{++}^{n}: \mathbf{p}^{\top} \mathbb{1}_{n}=m\right\}$. Finally, the tangent space of the simplex is defined as $\mathrm{T} \Delta=\left\{\mathbf{z} \in \mathbb{R}^{n}: \mathbf{z}^{\top} \mathbb{1}_{n}=0\right\}$.

Each energy unit is assigned to each actuator $i \in \mathcal{S}$ depending on the energy requirements. Furthermore, the energy required by the actuator $i \in \mathcal{S}$ is given by the function $F_{i}(\mathbf{p})$, whose mapping is given by $F: \Delta \mapsto \mathbb{R}$. Moreover, the vector of all the fitness functions is denoted by $\mathbf{F}(\mathbf{p}) \in \mathbb{R}^{n}$, whose mapping is given by $\mathbf{F}: \Delta \mapsto \mathbb{R}^{n}$.

The framework of the proposed methodology to manage a resource in a distributed way with a population dynamics approach is given by the use of stable and full potential games, which are defined next.

Definition 1: The game $\mathbf{F}(\mathbf{p})$ is stable if the Jacobian matrix $\mathbf{J}=D \mathbf{F}(\mathbf{p})$ is negative semi-definite with respect to the tangent space $\mathrm{T} \Delta$ [6], i.e.,

$$
\mathbf{z}^{\top} \mathbf{J} \mathbf{z} \leq 0, \text { for all } \mathbf{z} \in \mathrm{T} \Delta, \mathbf{p} \in \Delta .
$$

Then, it implies that a game is stable if the functions of energy requirements $F_{i}$, for all $i \in \mathcal{S}$, are decreasing with respect to the proportions $\mathbf{p} \in \Delta$.

Definition 2: The game $\mathbf{F}(\mathbf{p})$ is a full potential game if there exists a continuous differentiable function $f(\mathbf{p})$, known as potential function [9], satisfying that

$$
\frac{\partial f(\mathbf{p})}{\partial p_{i}}=F_{i}(\mathbf{p}), \text { for all } i \in \mathcal{S}, \mathbf{p} \in \Delta .
$$

Then, a full potential game is generated from a known potential differentiable function $f(\mathbf{p})$.

Notice that according to Definitions 1 and 2, the full potential game $\mathbf{F}(\mathbf{p})$, generated from a concave potential function $f(\mathbf{p})$, is stable. This is because the Jacobian matrix $\mathbf{J}$ corresponds to the Hessian matrix of the function $f(\mathbf{p})$. Moreover, consider the following optimization problem:

$$
\begin{aligned}
\underset{\mathbf{p}}{\operatorname{maximize}} & f(\mathbf{p}) \\
\text { subject to } \mathbf{p}^{\top} \mathbb{1}_{n} & =m \\
p_{i} & \geq 0, \text { for all } i \in \mathcal{S},
\end{aligned}
$$

where $f(\mathbf{p})$ is strictly concave, and the constraints (1b), and (1c) correspond to the simplex $\Delta$. In [9], it is shown that the solution of the potential game $\mathbf{F}(\mathbf{p})=\nabla f(\mathbf{p})$ corresponds to the solution of the optimization problem (1), since the Nash equilibrium of the population game satisfies the corresponding first-order Karush-Kuhn-Tucker (KKT) conditions.

\section{A. Distributed projection dynamics}

The traditional projection dynamics equation is one of the six fundamental population dynamics [9], and it requires full information (i.e., all actuators need information about all other actuator's energy requirements to evolve). However, the distributed projection dynamics are deduced in [4] from a local revision protocol that only needs partial information. Due to the fact that the local protocol is deduced from a non-well-mixed population, then there is an undirected non-complete connected graph describing possible local interaction denoted by $\mathcal{G}=(\mathcal{V}, \mathcal{E})$, where $\mathcal{V}$ is the set of nodes that represents the possible actuators (strategies) in the game, and $\mathcal{E} \subset\{(i, j): i, j \in \mathcal{V}\}$ is the set of links representing the information sharing within the system (communication network). Moreover, the deduction of the distributed projection dynamics can be found in [4], which are given by the following differential equation:

$$
\dot{p}_{i}=\left|\mathcal{N}_{i}\right| F_{i}(\mathbf{p})-\sum_{j \in \mathcal{N}_{i}} F_{j}(\mathbf{p}), \text { for all } i \in \mathcal{S},
$$

where $\mathcal{N}_{i}=\{j:(i, j) \in \mathcal{E}\}$ is the set of neighbors of the node $i \in \mathcal{V}$. Notice that $i \notin \mathcal{N}_{i}$, and that $\mathcal{N}_{i} \neq \emptyset$, for all $i \in \mathcal{V}$ since $\mathcal{G}$ is connected.

Besides, in [4] the stability analysis of the equilibrium point and the invariance feature of $\Delta$ under the distributed projection dynamics are formally presented.

\section{B. Distributed resource management approach}

As it has been mentioned in the previous section, it is supposed that the system is composed of $n$ sub-systems. Also consider that each sub-system $i \in \mathcal{S}$ is single-inputsigle-output (SISO), where $u_{i}$ is the control input and $y_{i}$ is the controlled output of the $i^{\text {th }}$ sub-system. Then, there is a 
controller for each sub-system computing a control action $u_{i}$ by using only local information, i.e., using information from the sub-system $i \in \mathcal{S}$, but not from sub-systems $\mathcal{S} \backslash\{i\}$.

Furthermore, there is an amount of energy $E_{i}$ necessary to establish the control action $u_{i}$ throughout an actuator, and it is given in energy units [e.u.]. For instance, if $u$ is a flow, $E$ would be the energy required by a valve or pump to establish the manipulated flow. For simplicity, a linear relation of the form $E_{i}=\phi_{i} u_{i}$ is considered in this paper. This conversion is made in order to treat the resource as an energy unit for all the controllers. Notice that the control actions for all the controllers in the system might be of different nature, i.e., it might be a flow, a voltage, a power, etc. Then, the conversion allows to translate all these signals of different nature into a unified unit, i.e., all the control signals given in the same unit in order to distribute optimally the total resource.

Consequently, the required energy to establish all the computed control actions is given by $\sum_{i \in \mathcal{S}} E_{i}$. However, suppose that the total available energy denoted by $m$ (population mass, see Table I) is not enough, i.e., $\sum_{i \in \mathcal{S}} E_{i}>m$.

The statement of the resource management optimization problem is as follows:

$$
\begin{aligned}
\underset{\mathbf{p}}{\operatorname{maximize}} f(\mathbf{p}) & =-\sum_{i \in \mathcal{S}}\left(E_{i}-p_{i}\right)^{2} \\
\text { subject to } \quad \mathbf{p}^{\top} \mathbb{1}_{n} & \leq m \\
p_{i} & \geq 0, \quad \text { for all } i \in \mathcal{S} .
\end{aligned}
$$

Notice that constraint ( $3 b$ ) can be expressed of the form of constraint (1b) by adding a slack variable [5]. Besides, due to the fact that the cost function (3a) is concave and differentiable, and according to Definitions 1 and 2, a stable full-potential game can be obtained. Then, the optimization problem (3) is solved in a distributed way by using the projection dynamics (2).

\section{WASTEWATER TREATMENT APPLICATION}

The proposed case study consists of three stages for the wastewater cycle as shown in Figure 1. First, it is considered an urban drainage system (UDS). At this stage, wastewater can be manipulated to avoid pollution. Then, wastewater flows to a treatment plant (WWTP) represented by a continuous stirred-tank reactor (CSTR) known as the catalytic ozonation pilot. After the wastewater is treated, it flows towards a storage tank to supply non-drinkable water demand for clean and city maintenance purposes, e.g., water for streets cleaning, plants watering, etc, in a non-drinkable water network (NDWN).

\section{A. Simplified Modeling}

Consider the catalytic ozonation pilot as a tank whose transversal area is given by $A_{1}=50 \mathrm{~m}^{2}$, whose inflow is given by the manipulated wastewater flow denoted by $q_{i, 1}$, and whose output is given by the treated water flow denoted by $q_{o, 1}$. Finally, let $h_{1}$ denote the level within the CSTR. Then, the dynamics for this stage are given by

$$
\dot{h}_{1}=\frac{1}{A_{1}}\left(q_{i, 1}-q_{o, 1}\right)
$$

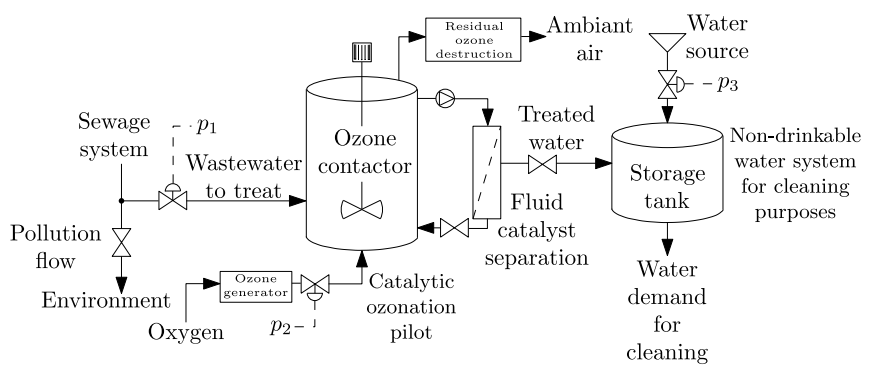

Fig. 1. Simplified scheme of the wastewater cycle, i.e., sewage system, WWTP (catalytic ozonation pilot), and non-drinkable water system. The process involves three different controllers whose control signals are given by $p_{1}, p_{2}$, and $p_{3}$.

where the outflow is expressed in terms of the current level by the Bernoulli's law, i.e., $q_{o, 1}=\kappa \sqrt{\rho g h_{1}}$, being $\rho=1.0204 \mathrm{~kg} / \mathrm{m}^{3}$ the density of the stored fluid, $g=9.8$ $\mathrm{m} / \mathrm{s}^{2}$ the gravity, and $\kappa=1$ for the current case study. The local control objective at this stage is to minimize the pollution. This objective is achieved by maintaining the WWTP working at its maximum capacity. Notice that when maintaining the WWTP working at the top, the inflow to it is maintained at the maximum possible value, reducing as much as possible the pollution flow (see Figure 1). Inflow $q_{i, 1}=u_{1}$ is controlled to maintain the CSTR at its maximum capacity level given by $r_{1}=5 \mathrm{~m}$. At this stage, there is a proportional-integral controller whose control law is given by

$$
u_{1}(t)=K_{p 1} e_{1}(t)+\int K_{i 1} e_{1}(\tau) d \tau,
$$

where $e_{1}(t)=r_{1}-y_{1}(t)$ is the error at the first controller. As a second stage, it is desired to control the ozone gas concentration at the top of the reactor denoted by $O z$ in order to treat the wastewater. The desired ozone gas concentration is denoted by $r_{2}=1.7 \mathrm{~mol} / 1$. This is achieved by manipulating the ozone generator power denoted by $u_{2}$. Moreover, the absorbance denoted by $A_{b}$ is the second state for the CSTR according to [1]. A simple state-space model of the catalytic ozonation pilot is

$$
\begin{aligned}
\dot{\mathbf{x}} & =\mathbf{A} \mathbf{x}+\mathbf{B} u_{2} \\
y & =\mathbf{C} \mathbf{x},
\end{aligned}
$$

where $\mathbf{x}=\left[\begin{array}{ll}O_{z} & A_{b}\end{array}\right]^{\top}$, and $y=O_{z}$. The matrices $\mathbf{A}, \mathbf{B}, \mathbf{C}$, and $\mathbf{D}$ are reported in [2], i.e.,

$$
\begin{aligned}
& \mathbf{A}=\left[\begin{array}{cc}
-0.0045 & 0 \\
0 & -0.00027
\end{array}\right], \quad \mathbf{B}=\left[\begin{array}{c}
0.00006 \\
-1.36 e-6
\end{array}\right], \\
& \mathbf{C}=\left[\begin{array}{ll}
1 & 0
\end{array}\right], \mathbf{D}=0 .
\end{aligned}
$$

At this stage, there is a proportional-integral controller whose control law is given by

$$
u_{2}(t)=-\mathbf{K} \mathbf{x}(t)+\int G e_{2}(\tau) d \tau,
$$

where $e_{2}(t)=r_{2}-y_{2}(t)$ is the error at the second controller. Finally, treated water flow $q_{o, 1}$ is transported to a nondrinkable water network (NDWN) represented by a storage 


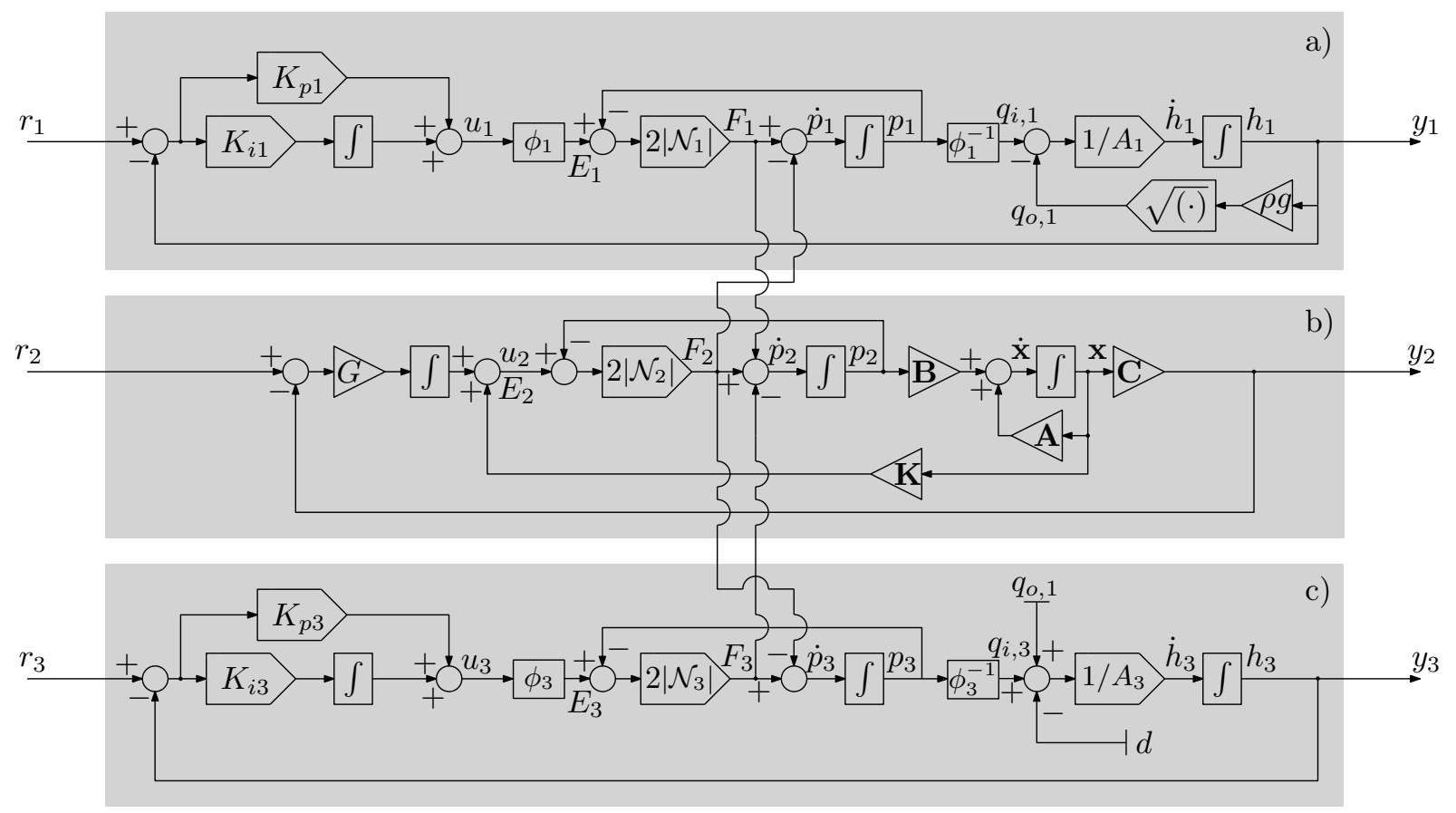

Fig. 2. Control scheme for the whole process: a) wastewater to treat control; b) catalytic ozonation pilot; and c) treated water control. The coupling between systems (a) and (c) through $q_{o, 1}$ is a dynamical coupling and it does not have anything to do with the information sharing for the distributed resource management strategy.

tank whose transversal area is given by $A_{3}=20 \mathrm{~m}^{2}$. The objective at this last stage is to maintain a safety level denoted by $r_{3}=10 \mathrm{~m}$ that is imposed by the company in charge of the management of the network, in order to satisfy a time-variant demand denoted by $d$ for clean-city maintenance. This control is made by manipulating a flow from an alternative water resource $q_{i, 3}=u_{3}$ (e.g., river, another WWTP, etc). The dynamics for this last stage are given by

$$
\dot{h}_{3}=\frac{1}{A_{3}}\left(q_{o, 1}+q_{i, 3}-d\right) .
$$

At this stage, there is a proportional-integral controller whose control law is given by

$$
u_{3}(t)=K_{p 3} e_{3}(t)+\int K_{i 3} e_{3}(\tau) d \tau,
$$

where $e_{3}(t)=r_{3}-y_{3}(t)$ is the error at the third controller. Furthermore, due to the fact that the proposed distributed controller manages the energy resource, it is necessary to compute the required energy in order to set the control actions, i.e., $E_{i}=\phi_{i} u_{i}$. For this particular example, the parameters to compute the required energy are given by $\phi_{1}=10, \phi_{2}=1$, and $\phi_{3}=10$. Finally, in order to emulate this time varying condition for cleaning purposes in the case study and illustrate the control performance again a disturbance, consider the following simple time-variant demand:

$$
d(t)= \begin{cases}20 \mathrm{~m}^{3} / \mathrm{min} & \text { if } t<576 \mathrm{~min} \\ 15 \mathrm{~m}^{3} / \mathrm{min} & \text { if } t \geq 576 \mathrm{~min}\end{cases}
$$

\section{B. Local controllers}

A general scheme for the proposed methodology with the distributed projection dynamics for the three stage WWTP application is shown in Figure 2. It is important to point out that this work focuses on the proper distributed resource management issue and that the design of the local controllers is out from scope of this paper. Consequently, the design of these classical controllers are not explained and just the tuning parameters are presented. Moreover, notice that the local control strategy does not affect the design of the proposed methodology since it only needs information about the control action $u_{i}$ and the associated required energy $E_{i}$ that the corresponding actuator requires to establish it. Then, any control strategy can be considered for the local subsystems. As an example, a proportional-integral controller is considered for the control of levels within the system (i.e., the level at the CSTR and at the storage tank), and a state feedback controller is considered for the ozone gas concentration regulation in the wastewater treatment process as in [2].

The control parameters used in this case study are shown in Table II corresponding to the control laws (4), (5), and (6).

Once the local controllers are designed, fitness functions for the distributed population dynamics are computed by using the corresponding control actions according to (3a). Then, the population game is solved in a distributed manner by using these functions, and with a communication graph given by $\mathcal{G}=(\mathcal{V}, \mathcal{E})$ that is presented in Figure 4, where $\mathcal{V}=\{1,2,3,4\}$, and $\mathcal{E}=\{(1,2),(2,3),(3,4)\}$. 


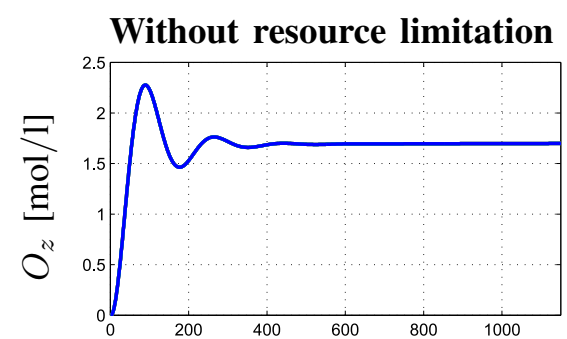

a)

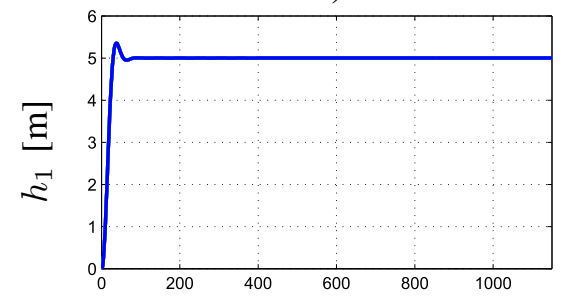

d)

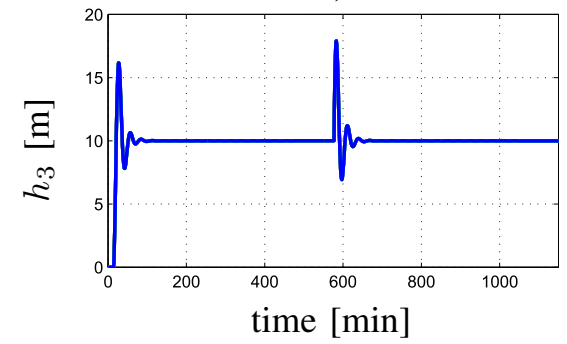

g)

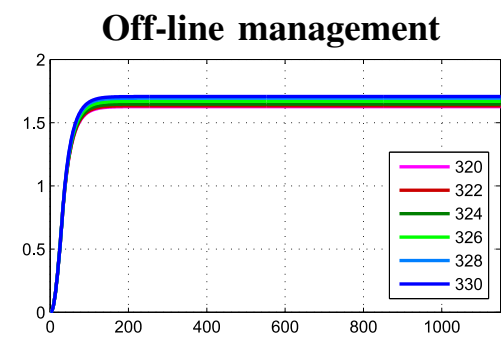

b)

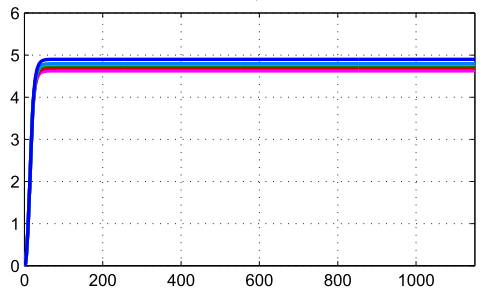

e)

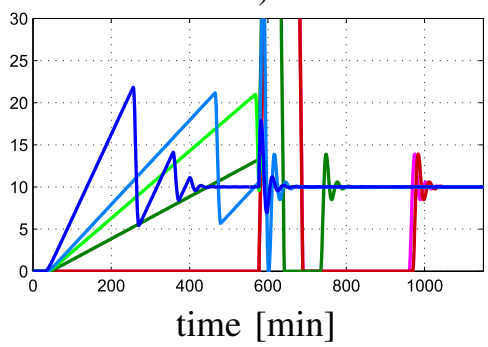

h)
On-line distributed management

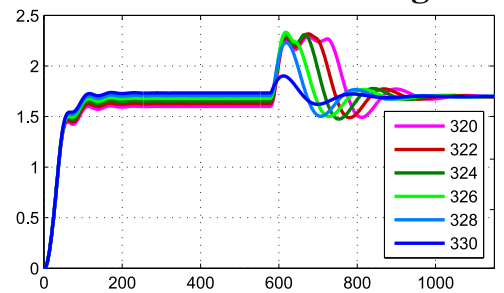

c)

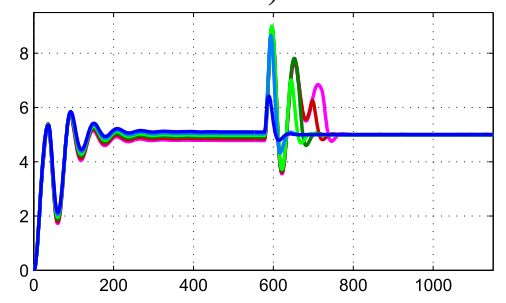

f)

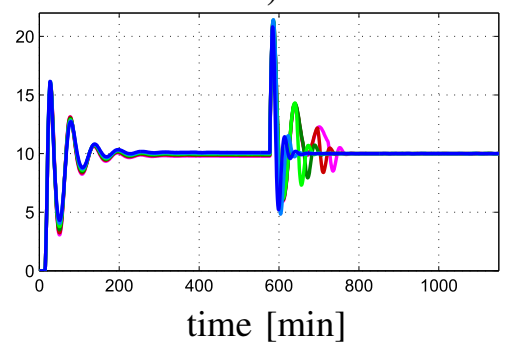

i)

Fig. 3. Comparison control results. Sub-figures show the control performance for ozone gas concentration $O_{z}$ and levels $h_{1}$, and $h_{3}$ as follows: a), $\mathrm{d}$ ), and g) for a control case without resource limitations; b), e) and h) for a control case with off-line management and limited energy resource of $320,322,324,326,328$, and 330 e.u.; and c), f), and i) for a control case with on-line distributed management (proposed approach) and limited energy resource of $320,322,324,326,328$, and 330 e.u.

TABLE II

CONTROL PARAMETERS FOR THE THREE LOCAL CONTROLLERS

\begin{tabular}{|c|c|}
\hline Control parameter & Value \\
\hline$K_{p 1}$ & 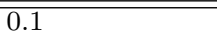 \\
\hline$K_{i 1}$ & 0.01 \\
\hline$G$ & 0.2345 \\
\hline $\mathbf{K}$ & $-\left[\begin{array}{ll}35.6590 & 42\end{array}\right]^{\top}$ \\
\hline$K_{p 3}$ & 0.3 \\
\hline$K_{i 3}$ & 0.01 \\
\hline
\end{tabular}

The node $1 \in \mathcal{V}$ is associated to the CSTR level problem, the node $2 \in \mathcal{V}$ is associated to the ozone gas concentration problem, the node $3 \in \mathcal{V}$ is associated to the storage tank level problem (see Figure 2), and the node $4 \in \mathcal{V}$ is associated to the required slack variable. Notice that the solution of the population game solves the optimization problem (3) subject to the energy constraint associated to the population mass $m$. Afterwards, the solution of the game is the final control action applied to each of the sub-systems.

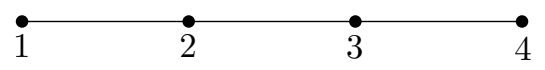

Fig. 4. Topology graph for the distributed resource management.

\section{RESULTS AND DISCUSSION}

The performance of the three controllers without any energy constraint is presented in Figures 3a), 3d), and $3 g$ ). Figure 3a) shows the evolution of the ozone gas concentration achieving the desired value for the wastewater treatment. Figure 3d) shows the evolution of the CSTR level achieving the established reference, and it is known that the pollution is reduced as the CSTR capacity is remained at its maximum value. Finally, Figure 3g) shows the storage tank level achieve the safety level to meet the time-variant demand. On the other hand, Figure 5a) shows the corresponding required energy, and it can be seen that the peak of required energy to achieve all the references is 389.2 e.u.

Suppose that the total available energy is lower than 389.2 e.u., then control actions should be saturated at convenient values. Figure $3 \mathrm{~b}$ ), $3 \mathrm{e}$ ), and $3 \mathrm{~h}$ ) show the evolution of the ozone gas concentration, the level at the CSTR, and the level at the storage tank, respectively, and for different limits for the available energy as presented in Figure 5b). Notice that the Figure 3h) exhibits a bad control performance when there is not enough energy to establish the proper control actions, i.e., the objective is not achieved for energy limitations of 320 , and 322. Furthermore, this undesired performance is compared with the control performance obtained with the proposed distributed methodology. 
Without resource limitation

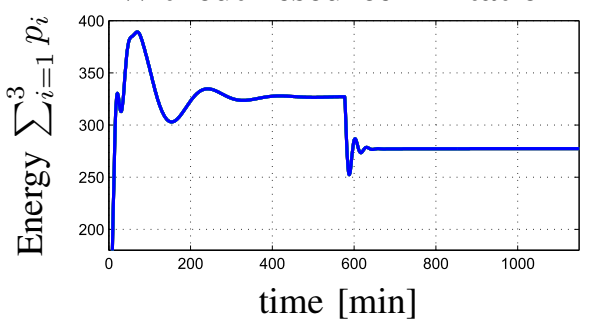

a)

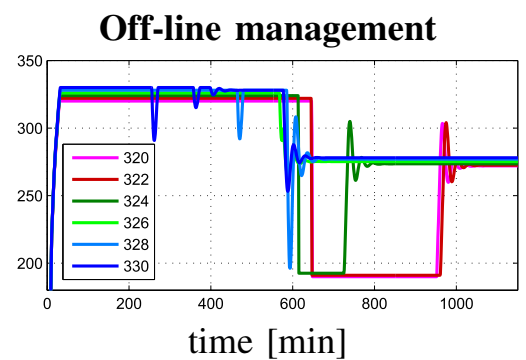

b)

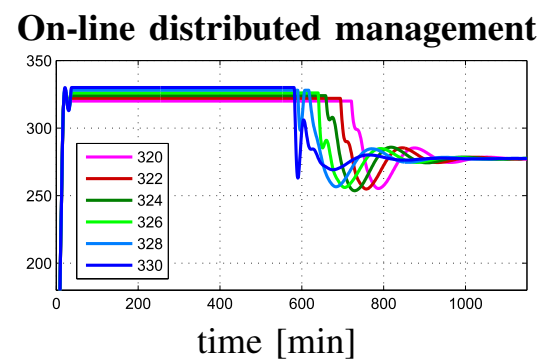

c)

Fig. 5. Total energy for the different control cases: a) control case without energy resource limitation $u_{i}=p_{i}, i=1, \ldots, 3$; b) control case with off-line management and limited energy resource of $320,322,324,326,328$, and 330 e.u; and c) control case with on-line distributed management (proposed approach) and limited energy resource of 320,322, 324, 326, 328, and 330 e.u.

Finally, Figures 3c), 3f), and 3i) show the evolution of the ozone gas concentration, the level at the CSTR, and the level at the storage tank, respectively, by applying the proposed distributed energy resource management based on the distributed projection dynamics. These results correspond to a limitation in the total energy shown in Figure 5c). Notice that the system behaves better than with an off-line energy management. This improvement can be clearly seen by comparing the evolution of the storage tank level, i.e., by comparing the control performance shown in $3 \mathrm{~h}$ ) and $3 \mathrm{i}$ ).

\section{CONCLUSIONS}

A control strategy to manage the available energy resource in a process is presented by using population dynamics. The strategy distributed in an on-line manner the resource by minimizing the difference between the computed control action by the local controllers, and the final applied energy to each sub-system. Moreover, it has been shown that this minimization problem satisfies the necessary conditions to obtain a stable full potential game. Besides, the performance of the controllers is significantly better than the control performance with an off-line energy management approach. This fact has been presented by mainly comparing the performance of the control level at the storage tank in the third stage of non-drinkable water. The better performance of the distributed resource management is obtained due to the fact that the resource is allocated throughout the controllers dynamically and equitably. In contrast, the offline management approach cannot distribute the resource in function of the requirements of the controllers.

\section{REFERENCES}

[1] M. Abouzlam, R. Ouvrard, D. Mehdi, F. Pontlevoy, B. Gombert, N.K. Vel Leitner, and S.O.B. Boukari. Identification and control of a wastewater treatment pilot by catalytic ozonation. In Proceedings of the American Control Conference (ACC), pages 6442 - 6447, 2012.

[2] M. Abouzlam, R. Ouvrard, D. Mehdi, F. Pontlevoy, B. Gombert, N.K. Vel Leitner, and S.O.B. Boukari. A robust control for wastewater treatment application. In Proceedings of the 3rd International Conference on Systems and Control (ICSC), pages 236 - 241, 2013.

[3] J. Barreiro-Gomez, G. Obando, C. Ocampo-Martinez, and N. Quijano. Making non-centralized a model predictive control scheme by using distributed smith dynamics. In Proceedings of the 5th IFAC Conference on Nonlinear Model Predictive Control (NMPC), 2015.

[4] J. Barreiro-Gomez, G. Obando, and N. Quijano. Distributed population dynamics: Optimization and control applications. IEEE Transactions on Systems, Man, and Cybernetics (Submitted), 2015.
[5] E.K.P. Chong and S.H. Zak. An Introduction to Optimization. Series in Discrete Mathematics and Optimization. Wiley, 2011.

[6] Josef Hofbauer and William H. Sandholm. Stable games and their dynamics. Journal of Economic Theory, 144(4):1665-1693, 2009.

[7] G. Obando, A. Pantoja, and N. Quijano. Building Temperature Control based on Population Dynamics. IEEE Transactions on Control Systems Technology., 22(1):404-412, 2014.

[8] A. Pantoja and N. Quijano. A population dynamics approach for the dispatch of distributed generators. IEEE Transactions on Industrial Electronics, 58(10):4559-4567, 2011.

[9] W. H. Sandholm. Population games and evolutionary dynamics. Economic learning and social evolution. Cambridge, Mass. MIT Press, 2010. 